\title{
Design and implementation of LQR/LQG strategies for oxygen stoichiometry control in PEM fuel cells based systems
}

\author{
Ali Niknezhadi, Miguel Allué-Fantova, Cristian Kunusch, Carlos Ocampo-Martínez* \\ Institut de Robòtica i Informàtica Industrial (CSIC-UPC) \\ Parc Tecnològic de Barcelona. C/ Llorens i Artigas 4-6, 08028 Barcelona, Spain.
}

\begin{abstract}
This paper presents the oxygen stoichiometry control problem of proton exchange Membrane (PEM) fuel cells and introduces a solution through an optimal control methodology. Based on the study of a non-linear dynamical model of a laboratory PEM fuel cell system and its associated components (air compressor, humidifiers, line heaters, valves, etc.), a control strategy for the oxygen stoichiometry regulation in the cathode line is designed and tested. From a linearized model of the system, an LQR/LQG controller is designed to give a solution to the stated control problem. Experimental results show the effectiveness of the proposed controllers design.
\end{abstract}

Keywords: LGQ/LQR control, PEM fuel cells, oxygen stoichiometry, experimental results.

\section{Introduction}

From the automatic control point of view, a fuel-cell-based (FCB) system is a nonlinear dynamic plant, with multiple inputs, multiple outputs, variables strongly coupled, model uncertainty and incidence of external disturbances. In this context, a topic that deserves special attention is the oxygen stoichiometry control $[1,2,3]$. If the oxygen flow at the cathode of a fuel cell is too low, it produces hot spots on the polymeric membrane, decrementing the cell power due to the lack of reactant in the triple contact areas. This phenomenon is known as cathode starvation and can lead to irreversible damages in the membranes [1,4]. On the other hand, an excessively high oxygen flow could cause a fast drying phenomenon of the polymeric membranes, increasing its ionic resistance. Apart from that, higher air flows mean more compressor power consumption, degrading the overall system energy conversion efficiency. Therefore, in order to avoid irreversible damages in the polymeric membranes and to obtain an efficient and reliable power response, it is necessary to design a control system capable of regulating the oxygen stoichiometry and thereby preserve proper operation conditions in the system.

According to preliminary works presented in $[1,5]$, the linear quadratic regulator (LQR) approach represents a promising option to start solving the stoichiometry control problem in PEM FCB systems with air compressors. In these first approaches, some initial assumptions such as ideal static humidifiers were taken into account. On the other hand, the LQR controller law is based on the availability of the complete state vector, which

\footnotetext{
${ }^{*}$ Corresponding author: Carlos Ocampo-Martínez Email: cocampo@iri.upc.edu Te: +34934015752
}

is not fully measurable in all cases. To overcome this inconvenient, an observer design based on three measurements (stack voltage, compressor air flow and manifold pressure) was developed to estimate the state vector. Finally, all the control tests were performed through simulations with the plant non-linear model.

The current control strategy, presented in this paper, resumes the idea firstly proposed by Pukrushpan et al using an LQR design for air regulation and system optimisation. Nevertheless, the non-linear model of the plant was completely validated in the test bench [6], the air humidifier was modelled as a dynamical system to include its slow response and a reduced-order Kalman observer based on single measurement was designed to estimate the two states needed for the control algorithm. Furthermore, the controller strategy was implemented and the parameters retuned in the fuel cells test bench [7].

Therefore, the main contributions of this paper lead to the application of LQR designs in the compressor air flow taking into account several scenarios related to variations in both the oxygen stoichiometry and the stack current. Although those designs were performed employing the linearisation of the validated mathematical model in [6], different controller set-ups have been stated and implemented over a real FCB system. Experimental results demonstrate the effectiveness of the control law for all the considered scenarios. Additionally, practical issues related to the implementation process and the fuel cell test bench are presented and discussed.

The reminder of this paper is organised as follows: In Section 2, a detailed description of the real FCB system is presented as well as the system model considered for controller designs. Section 3 briefly explains the LQR/LQG strategy and the considered control objectives. In Section 4 the most relevant results obtained from the implementation of the controller designs are 
presented and discussed. The main conclusions and some lines of further research are exposed in Section 5.

$\begin{array}{ll}\text { Nomenclature } \\ \text { System } & \\ & \\ m_{\text {hum }, c a} & \text { Mass of air in the cathode humidifier }[\mathrm{kg}] \\ m_{v_{2}, \text { an }} & \text { Mass of vapour in the stack anode }[\mathrm{kg}] \\ m_{v_{2}, c a} & \text { Mass of vapour in the stack cathode }[\mathrm{kg}] \\ m_{\mathrm{H}_{2}, \text { an }} & \text { Mass of hydrogen in the stack anode }[\mathrm{kg}] \\ m_{\mathrm{O}_{2}, c a} & \text { Mass of oxygen in the stack cathode }[\mathrm{kg}] \\ m_{N_{2}, c a} & \text { Mass of nitrogen in the stack cathode }[\mathrm{kg}] \\ n & \text { Number of cells in the stack } \\ I_{s t} & \text { Stack current [A] } \\ F & \text { Faraday constant [C/mol] } \\ M_{\mathrm{O}_{2}} & \text { Oxygen molar mass [kg/mol] } \\ V_{c p} & \text { Compressor DC voltage [V] } \\ P_{n e t} & \text { Output net power [W] } \\ W_{c p} & \text { Compressor air flow [kg/sec] } \\ W_{c p, r e f} & \text { Desired compressor air flow [kg/sec] } \\ W_{H_{2}} & \text { Cathode hydrogen flow [kg/sec] } \\ \lambda_{O_{2}} & \text { Cathode oxygen stoichiometry } \\ \lambda_{O_{2}, \text { des }} & \text { Desired cathode oxygen stoichiometry } \\ \omega_{c p} & \text { Compressor shaft angular speed [rad/sec] } \\ \chi O_{O_{2}} & \text { Molar fraction of oxygen in the air } \\ \Omega_{a t m} & \text { Relative humidity of the ambient air } \\ & \end{array}$

\section{Controller}

$f \quad$ Drift vector field

$g \quad$ Control vector field

$u \quad$ Control action of the non-linear system

$v \quad$ Control action of the linearised system

$v_{p} \quad$ Noise vector of the linearised system

$x \quad$ State vector of the non-linear system

$y \quad$ Output vector of the linearised system

$z \quad$ State vector of the linearised system

$\hat{z} \quad$ Observed state vector of the linearised system

$A \quad$ Linearised system matrix

$B \quad$ Linearised control matrix

$C \quad$ Linearised output matrix

$J \quad$ LQR cost function

$K_{r} \quad$ Kalman estimation gain

$K_{r} \quad$ State feedback gain

$Q \quad$ LQR design matrix

$R \quad$ LQR design matrix

$W_{p} \quad$ Linearised perturbation matrix

R2-

A

\section{PEM Fuel Cell Test Bench and Control Model}

\subsection{System under study}

The laboratory system under consideration is mainly composed by a fuel cell stack, an air compressor, a hydrogen storage tank, gases manifolds, humidifiers and line heaters. The

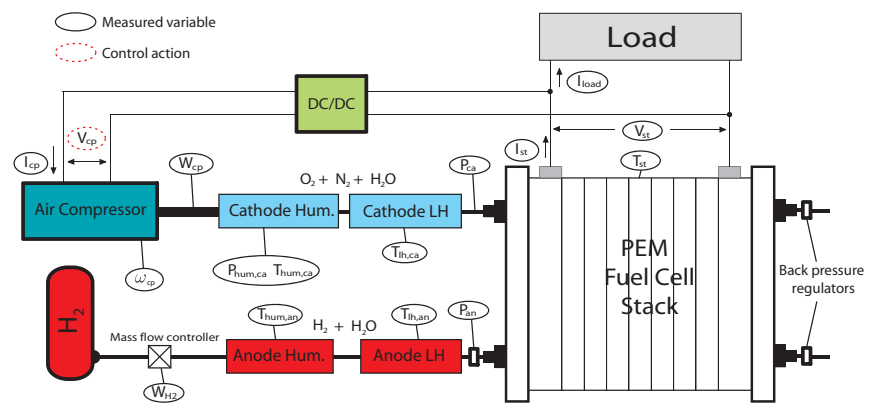

Figure 1: Schematic diagram of the experimental test station

schematic diagram in Figure 1 depicts the PEM fuel cell laboratory arrangement, Figure 2 shows the real test bench elements and Table 1 presents their description.

Some of the technical specifications of the laboratory fuel cell system under study are summarised as follows: the stack is an ElectroChem ${ }^{\circledR} 7$-cell stack with Nafion $115^{\circledR}$ membrane electrodes assemblies (MEAs), with a catalyst loading of 1 $\mathrm{mg} / \mathrm{cm}^{2}$ of platinum, $50 \mathrm{~cm}^{2}$ of active area, $50 \mathrm{~W}$ of nominal power and $100 \mathrm{~W}$ peak power. Cellkraft ${ }^{\circledR}$ membrane exchange humidifiers are used to maintain proper humidity conditions inside the cells, which is crucial to ensure the optimal operation of PEM membranes. The air compressor consists in a $12 \mathrm{~V} \mathrm{DC}$ oil-free diaphragm vacuum pump. The line heaters and stack temperatures are controlled by using a power station via decentralized PID controllers, allowing independent gas conditions (humidity and temperature) inside the stack.

Table 1: Description of the laboratory elements depicted in Figure 2

\begin{tabular}{cl}
\hline Element number & Description \\
\hline 1 & Electronic load \\
2 & Air compressor \\
3 & Stack temperature control unit \\
4 & Cathode back pressure valve \\
5 & Air/Oxygen humidifier \\
6 & Hydrogen humidifier \\
7 & PEM fuel cell stack \\
8 & Real time computer \\
9 & Stack heater \\
10 & Temperature sensor \\
11 & Current collector \\
12 & Bipolar plates \\
\hline
\end{tabular}

\subsection{Nonlinear model of the system}

Adopting a nonlinear control design approach, all the subsystems have been modelled and analyzed individually in order to obtain a nonlinear state-space model [6]. After regrouping the resultant differential equations, in conjunction with the static relationships between variables, the following description 


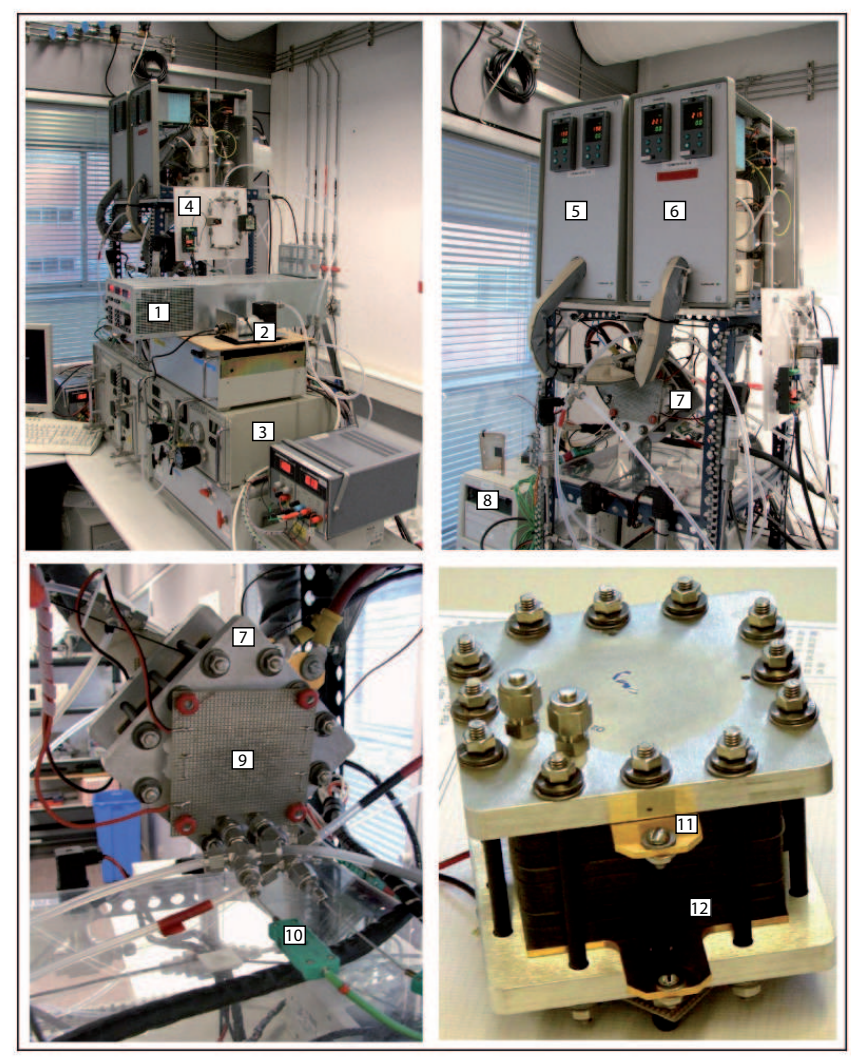

Figure 2: Fuel cell test bench at IRI (CSIC-UPC)

of seventh-order nonlinear control affine system was obtained:

$$
\frac{d x(t)}{d t}=f(x(t))+g(x(t)) u(t)
$$

where $x \in \mathbb{R}_{+}^{7}$ corresponds to the vector of the states variables and $u \in \mathbb{R}_{+}$denotes de control variable. The description of the state variables and the control action is outlined as follows:

$$
\begin{aligned}
& x_{1}=\omega_{c p}, \quad x_{2}=m_{h u m, c a}, \quad x_{3}=m_{O_{2}, c a}, \\
& x_{4}=m_{N_{2}, c a}, \quad x_{5}=m_{v_{2}, c a}, \quad x_{6}=m_{H_{2}, a n}, \\
& x_{7}=m_{v_{2}, a n}, \quad u=V_{c p} .
\end{aligned}
$$

\subsection{Model linearisation}

In order to design LQG controllers for the system under study, it is necessary to preform a model linearisation in a neighbourhood of a given working point. The linearisation procedure consists in expanding the right side of (1) in Taylor series around a stationary operating point and consider its first derivative (Jacobian matrix). The choice of the working point was based on practical considerations and in a way to get an average point within the operation range of the stack. The Taylor development of (1) around $x(0) \triangleq x_{0}$ at the first order in $z(t)=x(t)-x(0)$ is given by

$$
\begin{aligned}
\frac{d z(t)}{d t} & =\left[\frac{\partial f(x(t))}{\partial x(t)}\right]_{x(t)=x_{0}} z+\left[\frac{\partial g(x(t))}{\partial x(t)}\right]_{x(t)=x_{0}}\left(u(t)-u_{0}\right) \\
& =A z(t)+B v(t)
\end{aligned}
$$

where $u_{0} \triangleq u(0)$ and $v(t)=u(t)-u_{0}$. The working point selected in this paper was defined considering a compressor voltage $\left(V_{c p}=u_{0}\right)$ of $6 \mathrm{~V}$, a load current $\left(I_{s t}\right) 2 \mathrm{~A}$, anode and cathode pressure of 1 bar and a hydrogen flow $\left(W_{H_{2}}\right)$ of $2 \mathrm{slpm}$. This fact implies the following values for the elements of the initial condition vector:

$$
x_{0}=\left[\begin{array}{cc}
207.3136 \\
2.558 & 10^{-4} \\
4.1 & 10^{-4} \\
1.415 & 10^{-3} \\
1.862 & 10^{-4} \\
1.27 & 10^{-4} \\
1.844 & 10^{-4}
\end{array}\right] \text {. }
$$

\section{Controller Design}

\subsection{Control Objectives}

In the world of analysis and control of FCB systems, there are diverse control goals related to different operational aspects of the system, such as the maximisation of its effective life, the optimisation of its electric benefits, the minimization of its maintenance, etc. The approach proposed in this paper is focused on maximizing the output net power of the complete energy conversion system $\left(P_{n e t}\right)$, i.e., the difference of the electric power of the fuel cell and the air compressor consumption.

As proposed in previous works [1, 2, 8], this power can be maximized through the proper regulation of the oxygen stoichiometry at the fuel cell stack. This latter objective is, in turn, achieved by controlling the mass inflow of oxygen to the fuel cell cathode. This fact allows to maintain at any time an oxidizer flow that can be efficiently reduced close to the catalytic areas of the MEA and hence satisfying the power requirements of the system load, taking always into account the parasitic consumption of the air compressor. However, several issues are present in the experimental set-up from the control and instrumentation point of view. Those issues can be, among others, the inaccessibility of certain variables and measurement noise characteristic of the sensors.

In this context, the design and employment of control schemes based on Linear Quadratic Gaussian (LQG) laws are proposed as promising options in this framework, since these strategies collect simultaneously the improvement of the control objectives and the robust estimation of the system states. Therefore, this section proposes and discusses an LQG design oriented to the net power maximization of a FCB system.

\subsection{LQR Problem}

The proposed approach regards to the design of a LQR and its variant LQG due to the consideration of noises and disturbance of Gaussian spectral distribution affecting the closed loop. The 
main assumptions of this control strategy are the statements of a time-invariant linear system and stochastic noise models with known statistic properties. Thus, the continuous-time linear model of the system (1), obtained through a standard linearisation procedure (see subsection 2.3), can be written as

$$
\begin{aligned}
\frac{d z(t)}{d t} & =A z(t)+B v(t)+W_{p} I_{\mathrm{st}}(t), \\
y(t) & =C z(t)+v_{p},
\end{aligned}
$$

where $z \in \mathbb{R}^{n_{s}}$ is the state vector, $v \in \mathbb{R}^{m}$ denotes the vector of manipulated variables, $y \in \mathbb{R}^{q}$ is the vector of the system outputs, $I_{\mathrm{st}} \in \mathbb{R}^{p}$ is the disturbance vector, and $v_{p} \in \mathbb{R}^{q}$ corresponds to a vector containing the considered measurement noises. $A, B, W_{p}$ and $C$ are the system matrices of suitable dimensions. The control strategy aims to compute the set of optimal control actions $v(t)$ such as the following cost function is minimized:

$$
J=\lim _{\tau \rightarrow \infty} \frac{1}{\tau} \int_{0}^{\tau}\left[z(t)^{\mathrm{T}} Q z(t)+v(t)^{\mathrm{T}} R v(t)\right] d t,
$$

where the tuning matrices $Q$ and $R$ are properly selected, fulfilling the design conditions $Q=Q^{\mathrm{T}} \geq 0$ and $R=R^{\mathrm{T}}>=0$. These matrices are known as the input/state penalization matrices, respectively, which are in charge of the prioritization of either the state tracking or the control energy. The solution of the LQR problem consists in finding a stabilizing gain $K_{r}$ for the state feedback law

$$
v(t) \triangleq-K_{r} z(t)
$$

where $K_{r}$ acts as the gain matrix of the controller in the closedloop scheme (for further details please refer to [9]).

\subsection{State Estimation}

In order to compute matrix $K_{r}$, it is necessary to have available the states $z_{1}$ and $z_{2}$ (corresponding to the variables $\omega_{c p}$ and $m_{\text {hum }, \mathrm{c}}$ of the linearised model), feature that is not always possible. Therefore, it is necessary to design a reduced-order state observer from the measurement of the output variable $W_{c p}$. This auxiliary dynamic system estimates the trajectories of these two desired variables of the fuel cell system with an error and convergence rate given by the design parameter $K_{e}$, which corresponds with the estimation gain.

Thus, the control law (5) is computed as $v(t)=-K_{r} \hat{z}(t)$, where $\hat{z}(t)$ is the reduced state estimate vector from the observer. Notice that the controller and the observer designs can be done independently (separation principle). On the other hand, in order to improve the closed-loop performance, and specifically to eliminate the steady-state regulation error, an integrator is usually added. Hence, the design of the LQR control law implies computing a gain $K_{r}$ considering the extended dynamic system [9].

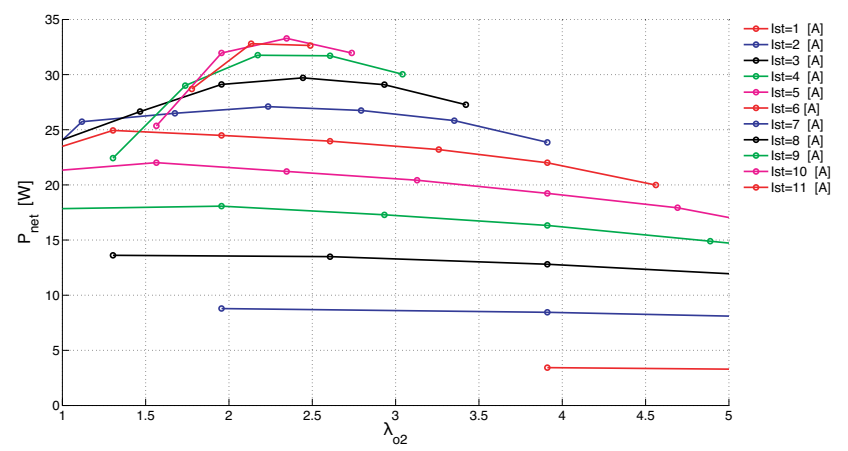

Figure 3: System energy conversion efficiency for different load conditions $\left(P_{n e t}\right.$ vs. $\left.\lambda_{O_{2}}\right)$

\section{Results and Discussion}

As formerly introduced, the pursued control objective of this regulation strategy is the optimization of the energy conversion of the FCB system, maximizing the net power generated by the system $\left(P_{n e t}\right)$ under different load conditions. In Figure 3 , it can be appreciated that accomplishing such optimal working condition is equivalent to maintain the cathode line oxygen stoichiometry $\left(\lambda_{\mathrm{O}_{2}}\right)$ close to an optimal value. This optimal value can be determined from a thorough off-line analysis of the open-loop system, considering changes in the current drained from the stack and a wide set of stoichiometry values (Figure $3)$. In an air-flow control scheme, the expression of the compressor air reference can be readily obtained from the desired value of the oxygen stoichiometry $\left(\lambda_{\mathrm{O}_{2} \text {,des }}\right)$. Given that the molar fraction of oxygen in the air $\left(\chi_{\mathrm{O}_{2}}\right)$ is a known parameter and taking into account a constant relative humidity of the ambient air $\left(\Omega_{a t m}\right)$, the desired mass flow of air $\left(W_{c p, r e f}\right)$ can be directly computed from the following equation:

$$
W_{c p, r e f}=\left(1+\Omega_{a t m}\right) \frac{1}{\chi O_{2}} \lambda_{O_{2}, d e s} M_{O_{2}} \frac{n I_{s t}}{4 F}
$$

where $n$ is the number of cells in the stack, $M_{O_{2}}$ the oxygen molar mass and $F$ de Faraday constant.

\subsection{Controller set-up}

The selection of the matrices pair Q-R for the final controller was done following an iterative process, starting from the simulations design and then adjusting the parameters in order to fit them to experimental constraints. The results of one of the best selections, from the performance point of view, are presented in the next subsection. As discussed in Section 3, the system state vector has been expanded with an integrator in order to eliminate the steady-state error of the air flow regulation. Besides, a classical anti-windup structure has been added to prevent the winding of the integrator when saturation occurs in the compressor actuator. In Figure 4, the implemented controller structure is depicted.

\subsection{Experimental results}

The objective of this subsection is to demonstrate the performance of the proposed LQG control strategy in the real operat- 


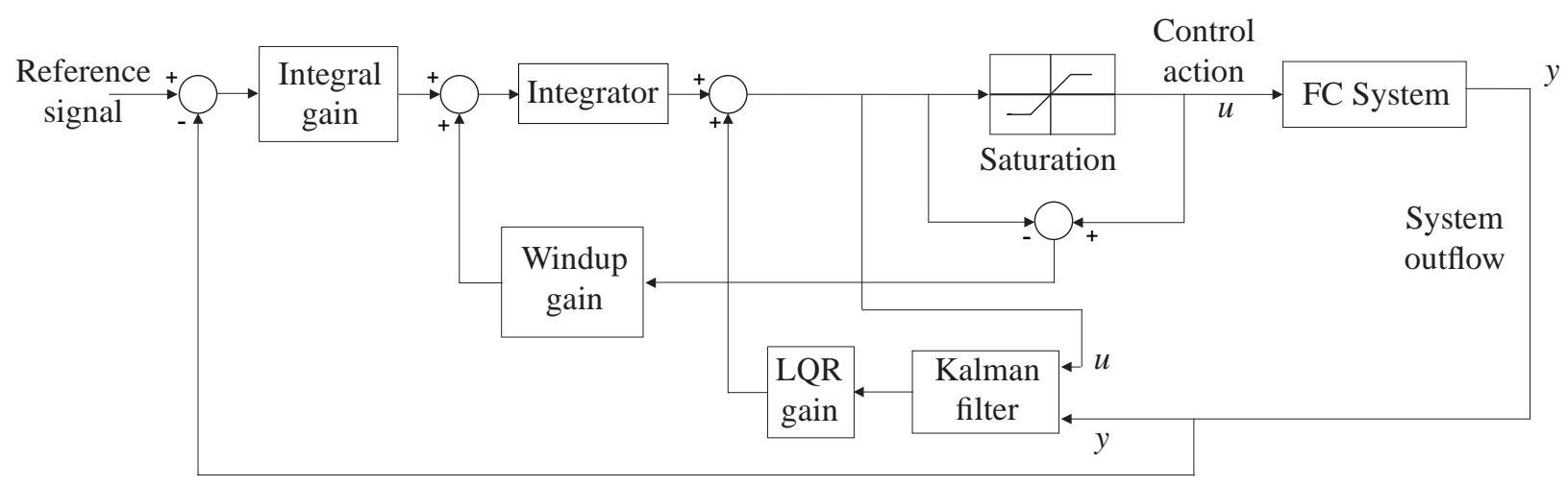

Figure 4: Schematic diagram of the controller structure

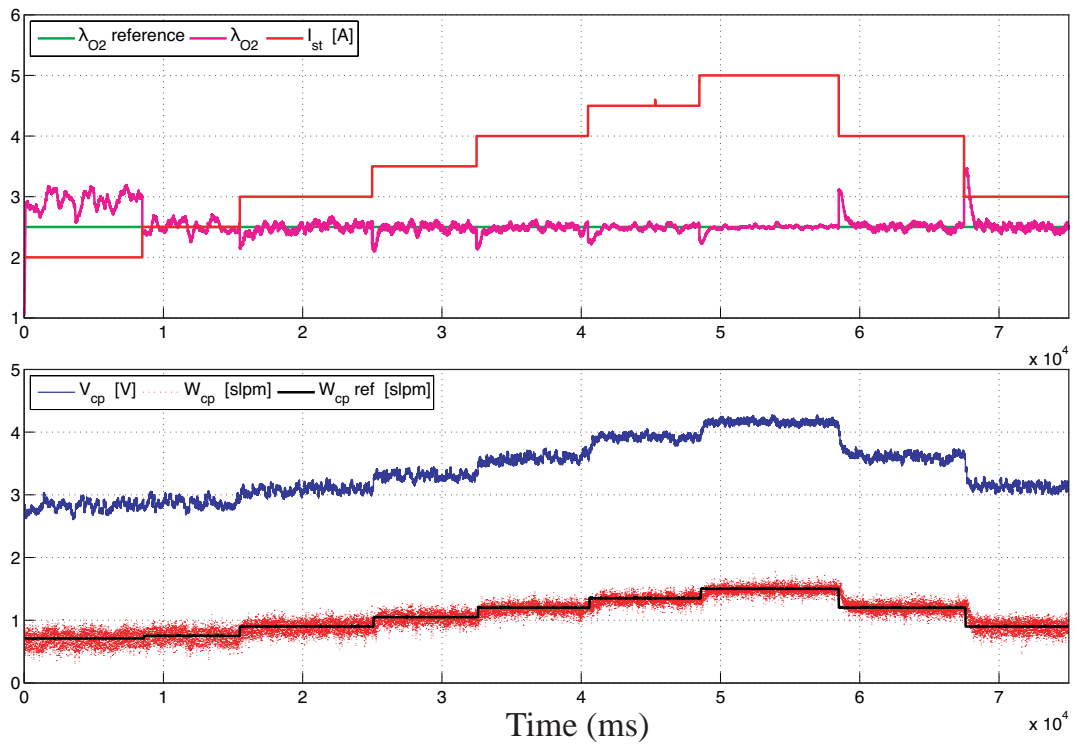

Figure 5: $\lambda_{O_{2}}$ regulation for variable load conditions

ing conditions and point out some considerations about practical issues. The first series of tests was performed considering a fixed oxygen stoichiometry and changes in the stack current (Figure 5). It is interesting to observe that, during the first eight seconds of the test, the control action was saturated in its lowest value, but when stack current changes to $2.5 \mathrm{~A}$ the controller recovers its regular behaviour by means of the anti-windup algorithm. Next, the oxygen stoichiometry can rapidly start tracking its reference. During the rest of the test, it can be noticed the regulation behaviour of the loop at different stack currents and the dynamic disturbance rejection ability of the system. Figures 5 and 6 exhibit the stack current variation $\left(I_{s t}\right)$, the oxygen stoichiometry $\left(\lambda_{O_{2}}\right)$ and its reference $\left(\lambda_{O_{2}, r e f}\right)$, the motor voltage $\left(V_{c p}\right)$, the compressor air flow $\left(W_{c p}\right)$ and the internal reference calculated by the controller $\left(W_{c p, r e f}\right)$.

Afterwards, a different set of tests was performed in the PEM fuel cell test station, considering a constant load current and oxygen stoichiometry reference variations. In Figure 6 , the control performance is shown when the internal variable $\lambda_{O_{2}}$ is regulated in a wide range of values.
The theoretical analysis and the experimental results show that the adopted control strategy stabilises the system around the working point and endows an improved dynamic behaviour to the closed loop. An important practical issue that needs to be carefully considered is the integrator gain tuning. Indeed, a choice of a high value ensures a fast response while causing permanent oscillations around the steady-state operation of the compressor. On the other hand, a low value ensures smooth response in steady-state operation, but presents degradation in the transient response. This compromise can be noticed experimentally.

\section{Conclusions}

In this paper, a linear control approach that solves an important control problem of PEM FCB generation systems is presented. The control law is based on the proper design of an LQR/LQG strategy. Such controller allows maximizing the energy conversion efficiency of the overall system and avoids the 

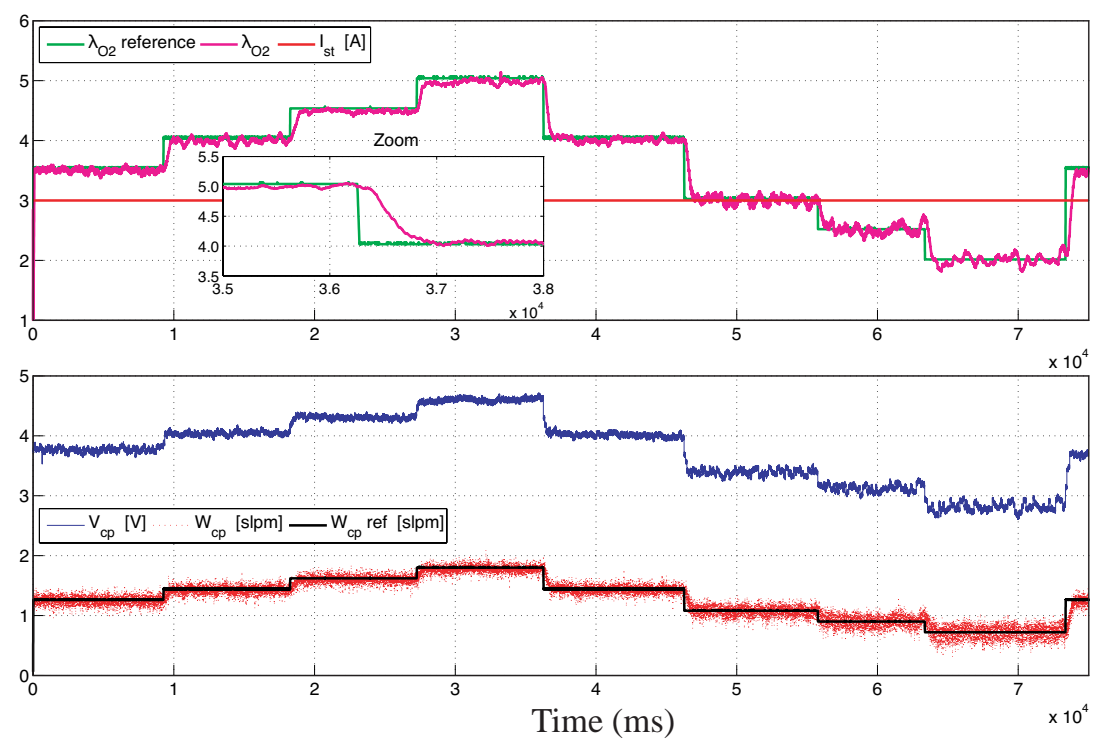

Figure 6: $\lambda_{O_{2}}$ regulation for constant $I_{s t}$

oxygen starvation problem. The designed control strategy efficiently regulates the stack oxygen stoichiometry and requires reduced information to operate since its law only depends on two easily measured variables $\left(W_{c p}\right.$ and $\left.I_{s t}\right)$. The control design is based on a linear model of the plant, but its analysis and validation is performed through experimental tests for different load conditions. The experimental results show the effectiveness and feasibility of the proposed control approach given real scenarios of both oxygen stoichiometry and stack current variations.

Currently, further research is being performed by the research group in the fuel cells control field, tackling nonlinear control solutions (e.g. higher order sliding modes and model predictive control), different control problems (e.g. membrane water transport and temperature control) and other plant configurations (e.g. open cathode fuel cells, self-humidified systems and hybrid power generation plants).

\section{Acknowledgments}

All the experimental tests were performed at the Fuel Cells Laboratory of the Institut de Robòtica $i$ Informàtica Industrial (CSIC-UPC, Barcelona) and only possible due to its advanced equipment and proficient technical staff. This research has been supported by the CICYT project DIP2007-62966, the Agencia Española de Cooperación Internacional (AECI) under the project A/026279/09, the Juan de la Cierva Research Programme (ref. JCI-2008-2438) and the CSIC JAE-DOC Research Programme (ref. NRP 734635NX0SC000087).

\section{References}

[1] J.T. Pukrushpan, A.G. Stefanopoulou, and H. Peng. Control of Fuel Cell Power Systems. Springer, 2004.
[2] C. Kunusch, P.F. Puleston, M.A. Mayosky, and J. Riera. Sliding mode strategy for PEM fuel cells stacks breathing control using a Super-Twisting algorithm. IEEE Transactions on Control System Technology, 17:167-174, 2009.

[3] R. Talj, M. Hilairet, and R. Ortega. Second order sliding mode control of the moto-compressor of a PEM fuel cell air feeding system, with experimental validation. In IECON '09. 35th Annual Conference of IEEE, 2009.

[4] F. Barbir. PEM fuel cells: theory and practice. Elsevier, 2005.

[5] J.T. Pukrushpan, A.G. Stefanopoulou, and H. Peng. Control of fuel cell breathing. IEEE Control Systems Magazine, 24(2):30-46, April 2004.

[6] C. Kunusch. Modelling and Nonlinear Control of PEM Fuel Cell Systems. PhD thesis, Electrical Department, National University of La Plata, September 2009.

[7] Fuel Cells Laboratory. Institut de Robòtica i Informàtica Industrial (CSICUPC), 2010. http://wikiri.upc.es/index.php/Fuel_Cell_Lab.

[8] C. Kunusch, P.F. Puleston, M.A. Mayosky, and M. Serra. Advances in HOSM control design and implementation for PEM fuel cell systems. In 14th IFAC International Conference on Methods and Models in Automation and Robotics, Miedzyzdroje, Poland., August 2009.

[9] S. Skogestad and I. Postlethwaite. Multivariable feedback control: analysis and design. Wiley New York, 2005. 